Chapman University

Chapman University Digital Commons

2019

Track 1.b Introduction: Re-Designing Health: Transforming

Systems, Practices and Care

Aidan Rowe

Claudine Jaenichen

Gillian Harvey

Kate Sellen

Stephanie Vandenberg

Follow this and additional works at: https://digitalcommons.chapman.edu/art_articles

Part of the Art and Design Commons, Health and Medical Administration Commons, and the Other Medicine and Health Sciences Commons 


\section{Track 1.b Introduction: Re-Designing Health: Transforming Systems, Practices and Care}

\section{Comments}

This article was originally published in Conference Proceedings of the Academy for Design Innovation Management, volume 2, issue 1, in 2019. https://doi.org/10.33114/adim.2019.1b

\section{Creative Commons License}

\section{(c) (1) $(9)$}

This work is licensed under a Creative Commons Attribution-Noncommercial-Share Alike 4.0 License.

\section{Copyright}

Conference proceedings of the Academy for Design Innovation Management 


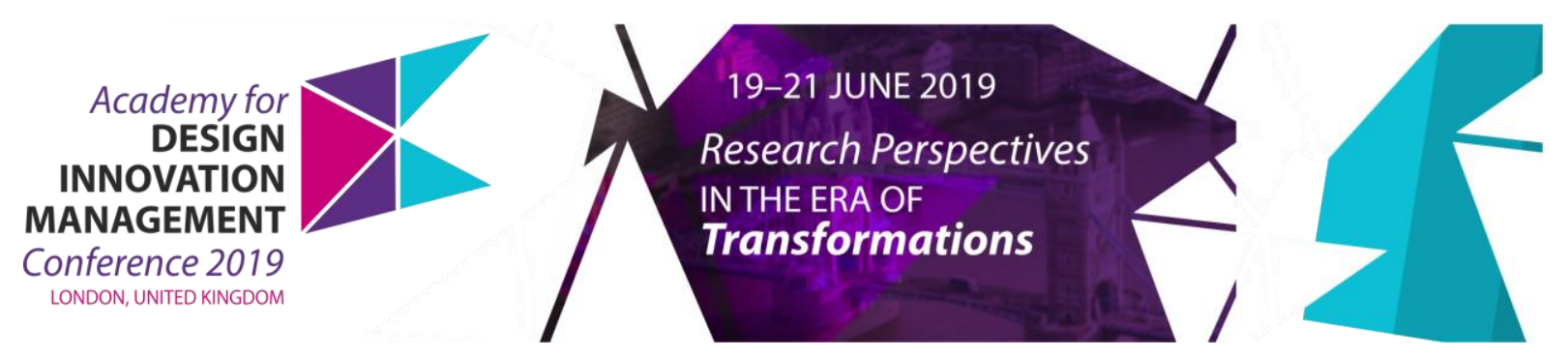

\title{
Track 1.b Introduction: Re-Designing Health: Transforming Systems, Practices and Care
}

\author{
ROWE Aidan ${ }^{a}$; JAENICHEN Claudine ${ }^{\mathrm{b}}$; HARVEY Gillian ${ }^{\mathrm{a}}$; SELLEN Kate ${ }^{\mathrm{c}}$ and VANDENBERG Stephanie ${ }^{\mathrm{d}}$ \\ a University of Alberta, Canada \\ ${ }^{\mathrm{b}}$ Chapman University, USA \\ c OCADU, Canada \\ d University of Calgary, Canada \\ doi: 10.33114/adim.2019.1b
}

The Re-Designing Health: Transforming Systems, Practices and Care track explores the increasing role and possibility for a wide range of design practices and methods to contribute to health care products, provision, and systems.

There is growing recognition of the increasing complexity faced by healthcare systems; critical issues and challenges include ageing populations, chronic diseases, growing drug ineffectiveness, and lack of access to comprehensive services (to name only a few examples). Concurrently design thinking, methods and practices are increasingly recognized as means of addressing complex, multi-levelled and systemic problems.

The track session brought together design academics, researchers and practitioners that are working in-and across - areas of design, medicine and health. Employing design methods, practices, and thinking to address a range of healthcare challenges - from individual product to large-scale policy. This track provided a forum for researchers, practitioners, students, and designers to provide evidence for these relationships, document challenges and successes and to provide theoretical and practical models for healthcare and design to work collaboratively to address complex healthcare problems.

We looked to identify and build research capacity to help address the complex and significant challenges faced by society in the 21st century and to chart new opportunities for the discipline of design.

This Re-Designing Health: Transforming Systems, Practices and Care track contains six papers that deal broadly with notions of design and health and document a variety of practices, proposals and ideas.

This Re-Designing Health: Transforming Systems, Practices and Care track contains six papers that deal broadly with notions of design and health and document a variety of practices, proposals and ideas.

Re-Designing Health: Transforming Systems, Practices and Care Track Chairs

Aidan Rowe, University of Alberta, Canada

Claudine Jaenichen, Chapman University, USA

Gillian Harvey, University of Alberta, Canada

Kate Sellen, OCADU, Canada

Stephanie VandenBerg, University of Calgary, Canada

In our first paper, Reframing Healthcare: Emerging Health Design Opportunities, design researchers, Aidan Rowe and Michelle Knox, explore the possibilities that design offers-methods, practices and processes-to help address the identified rising complications related to contemporary healthcare provision. 
While design and health have a long history of working together, much of this work has been limited. In this paper, the authors make the case for further opportunities for design and health to work together in deep, meaningful and human ways.

They begin by discussing the changing space of design, then articulate the similarities between design and healthcare. The authors then present two health design research projects that employ design methods and processes within healthcare settings, exploring new opportunities for design and health to collaborate. They conclude by summarizing the benefits and challenges of these projects, articulating future possibilities for design and healthcare to collaborate.

In the track's second contribution-Aesthetic Considerations in the Ortho-Prosthetic Design Process-Yan Luximon, Parth Shah, and Hassan Iftikhar explore the role and possibility of applying design to the existing functional process of prosthetic creation.

They note that medical products, including prosthetics and orthotics, are designed to partially or completely assist or replace the functionality of specific body parts affected by ailments or medical deformities. People using such devices share similar sensibilities and concerns, such as looking attractive or being able to wear fashionable clothing. However, due to a greater emphasis on function over fashion in designing these medical products, the aesthetic values of the user are not fully considered. This aesthetic paucity may have a strong psychological and cognitive impact, which affects the user experience. Hence, this study aims to explore key parameters affecting the aesthetics of medical products such as prosthetics and orthotics and identify the challenges involved in their design process. Recommendations have also been suggested for the designers with the help of a design example.

Looking at a framework level researchers Jessica Lea Dunn, Keum Hee Kimmi Ko, David Lahoud, Erez Nusem, Karla Straker, and Cara Wrigley explore design innovation and medical devices in Exploring the Role of Design in the Context of Medical Device Innovation.

In this third paper the authors recognize that technology is the leading driving force in healthcare and medical device design, however, innovations which emerge from these practices are often driven by clinical requirements. Such innovations are focused on developing products that address current health issues, diseases or medical problems - often lacking consideration of the end-users' needs.

Design innovation advocates that user-centred design happens much earlier in the product development process so that the patient needs are prioritised. However, this emerging field is yet to be defined and explored in a medical context. This paper, therefore, proposes a framework of Medical Device Design Innovation to explore the role of design in medical device innovation through two medical device case studies. The proposed framework suggests a way to navigate the nuances and complexities of the medical device industry in order to put the patient first while ensuring commercial viability.

In A Collaboration of University and Civil Society Organisation: Development of a Web-Based Platform for Promoting Accessibility in Design authors Abdusselam Selami Cifter, Ramazan Bas, and Sema Ergonul explore the intersection of inclusive design, collaboration and design.

They state that accessibility is a fundamental element and a basic requirement of our daily lives; however, in many cases, we are not aware of it unless we encounter its absence. Particularly people with disabilities regularly experience accessibility problems in various domains, wherein certain cases these problems even hinder them from accessing their basic needs.

Designers have an important responsibility to design inclusively; however, they need a diverse range of reliable and up-to-date information for this, which also fits with their requirements. This paper presents an example of a collaboration of university-civil society organization within the scope of "I CAN ACCESS" project, which aimed to develop a web-based platform for designers' use in Turkey in an effort to increase their awareness on accessibility and assist them to design inclusively. The paper particularly focuses on the advantages of collaborations between civil society organizations and universities by revealing their specific resources and possibilities.

In the track's fifth paper-Gaining Patient Experience Insights: An Integrated and Multi-Leveled Framework of Information-designer researchers Maitane Garcia-Lopez, Ester Val, Ion Iriarte, Raquel Olarte, and Marina Gonzalez-Zubiaurre explore the lived experiences of health care users. 
Taking the patient experience as a basis, this paper introduces a theoretical framework, to capture insights leading to new technological healthcare solutions. Targeting a recently diagnosed type 1 diabetes child and her mother (the principal caregiver), the framework showed its potential with effective identification of meaningful insights in a generative session. The framework is based on the patient experience across the continuum of care. It identifies insights from the patient perspective: capturing patients' emotional and cognitive responses, understanding agents involved in patient experience, uncovering pain moments, identifying their root causes, and/or prioritizing actions for improvement.

The framework deepens understanding of the patient experience by providing an integrated and multi-levelled structure to assist designers to (a) empathize with the patient and the caregiver throughout the continuum of care, (b) understand the interdependencies around the patient and different agents and (c) reveal insights at the interaction level.

In the final paper in our track, Design As An Agent For Public Policy Innovation, authors Federico Vaz and Sharon Prendeville explore the intersection of public policy, innovation, design and the rise of policy labs.

Described as units developing public policies in a design-oriented manner, Policy Labs are tasked to innovate to gain in policy effectiveness and efficiency. However, as public policymaking is a context-dependent activity, the way in which these novel organizations operate significantly differs. This study discusses the emergence of design approaches for policy innovation. The purpose is to map how Policy Labs in Europe introduce design approaches at distinct stages of the policymaking cycle.

For this study, 30 organizations in Europe operating at various levels of government were surveyed. Based on the public policymaking process model, it investigates which design methods are Policy Labs deploying to innovate public policies. The study exposed a gap in the awareness of the utilized methods' nature. It also showed that the use of design methods is of less importance than the introduction of design mindsets for public policy innovation, namely 'user-centredness', 'co-creation', and 'exploration'.

The collected papers in our track-Re-Designing Health: Transforming Systems, Practices and Care-recognize the power and possibility for design to contribute to $21^{\text {st }}$-century healthcare products, provision, and systems. From higher-level frameworks to diagrammatizing patient experience to specific case studies of practice, the exciting range of research demonstrated articulates how design is addressing the multifaceted and substantial healthcare challenges faced today. 